\title{
STABLE LINEAR FRACTIONAL TRANSFORMATIONS WITH APPLICATIONS TO STABILIZATION AND MULTISTAGE $H^{\infty}$ CONTROL DESIGN
}

\author{
WEI-YONG YAN \\ School of Electrical and Electronic Engineering, Nanyang Technological University, Nanyang Ave., Singapore 2263 \\ AND \\ JOHN B. MOORE \\ Department of Systems Engineering, Research School of Physical Sciences and Engineering, Australian National \\ University, GPO Box 4, Canberra, ACT 2601, Australia
}

\begin{abstract}
SUMMARY
Stable linear fractional transformations (SLFTs) resulting from a $2 \times 2$-block unit $Z$ in the ring of stable real rational proper matrices are considered in this paper. Several general properties are obtained, including properties with respect to possible pole-zero cancellations and a generic McMillan degree relationship between a transfer matrix and its image under an SLFT. The problem of representing a plant as an SLFT of another plant such that the order of the original plant is exactly equal to the sums of the orders of the SLFT and of the new plant is solved. All such representations can be found by searching for all matching pairs of stable invariant subspaces associated with the plant.

In relation to applications of SLFTs, it is shown that if two plants are related by an SLFT, then a one-toone correspondence between their two respective sets of all stabilizing controllers can be established via a different SLFT. Also, it is shown how to decompose a standard $H^{\infty}$ control problem by means of SLFT into two individual $H^{\infty}$ subproblems, the first involving a nominal plant model and the second involving a certain frequency-shaped approximation error. An example is presented to illustrate the idea of decomposing the complexity of an $H^{\infty}$ control problem.
\end{abstract}

KEY WORDS rational matrices; fractional linear transformation; stabilization; $H^{\infty}$ control

\section{INTRODUCTION}

Linear fractional transformations (LFTs), defined via a unit in the ring of stable proper rational matrices denoted by $R H_{\infty}$, have been found to arise frequently in systems theory, see, for example, References $11,13,5,9$ and 17. As is well known, any particular stabilizing compensator for a plant is an image of a suitable parameter $Q$ in $R H_{\infty}$ by a fixed LFT defined via a unit in $R H_{\infty}$, and vice versa. By duality, any plant which is stabilized by a compensator can

\footnotetext{
"Work partially supported by Boeing (BCAC) and DSTO Australia.
}

This paper was recommended for publication by editor D. Limebeer

CCC 1049-8923/96/020101-22

(C) 1996 by John Wiley \& Sons, Ltd.
Received 12 September 1991

Revised 1 July 1994 
also be viewed by an LFT as an image of a parameter $S$ in $R H_{\infty}$. In fact, any plant can be expressed as a stable linear fractional transformation of a certain frequency-shaped mismatch between the plant and its given model. Recently, Tay et al. ${ }^{15}$ proposed a control scheme in which the mismatch can be identified on-line from measurement data and illustrated that in a robust controller design context there could be an advantage of identifying the mismatch instead of the plant in respect of model reduction.

LFTs defined via a unit in the ring of polynomial matrices have been considered by Antoulas ${ }^{1}$ from the standpoint of realization. It is shown that every system can be decomposed into a number of subsystems via a series of LFTs subject to certain realization constraints. This decomposition has two nice properties in respect of McMillan degree and Kronecker index. However, since the LFT in Reference 1 is defined based on polynomial coprime factorizations, it may be difficult to apply the decomposition to a synthesis problem in spite of its attractive properties. In addition, it is not clear whether the decomposition possesses any interesting topological properties.

This paper is concerned with an LFT induced by a $2 \times 2$-block unit in $R H_{\infty}$. Such a stable LFT (hereafter called an SLFT) is a transformation from one system to another and more precisely a bijective mapping from $R_{\mathrm{p}}$ onto itself. Though $R_{\mathrm{p}}$ is not a Hilbert space, it can be equipped with the graph topology introduced by Vidyasagar. ${ }^{17}$ With this topology, it turns out that an SLFT is continuous.

Our objective is to study some basic properties of an SLFT from a system point of view and to explore application of SLFT in robust stabilization and $H^{\infty}$ controller design. For example, we derive properties with respect to possible pole-zero cancellations and a generic McMillan degree relationship between a transfer matrix and its image under an SLFT in the state-space sense. If two plants are related by an SLFT, then a one-to-one correspondence between their two respective sets of all stabilizing controllers is established via a different SLFT. This suggests a two-step procedure of designing a stabilizing controller for a high-order plant expressed as a stable linear fractional transformation of some frequency-shaped modelling error - one step designing a stabilizing controller for the plant model and the other enhancing this controller to cope with the high-order plant. An advantage of this method is avoiding directly designing a high-order controller for some class of plants of high order. Another important application of SLFT is in $H^{\infty}$ controller design. Specifically, a standard $H^{\infty}$ control problem can be approximately decomposed by means of SLFT into two individual $H^{\infty}$-subproblems, the first involving a nominal plant model and the second involving a certain frequency-shaped approximation error. This application is of particular significance when there exist unmodelled dynamics or the order of a plant is so high that it might be infeasible to solve directly the original problem. Moreover, the theory would support an approach when the first $H^{\infty}$ design is off-line and the second 'on-line' based on some on-line identification. An example presented in the paper shows that the idea of decomposing the complexity of an $H^{\infty}$ problem works very well and that a satisfying $H^{\infty}$ controller of order much lower than the order of the true plant can be designed by using a low-order balanced truncation approximation of the generalized frequencyshaped modelling error.

The application of SLFTs in breaking a design problem into two subproblems with less complexity offers one an incentive to ask a question as to whether a plant can be minimally represented as an SLFT of another plant in the sense that the complexity (order) of the given plant is exactly the sum of the complexities of the SLFT and of the new plant. This question is addressed in this paper and a complete answer is obtained in terms of stable invariant subspaces.

The next section will be devoted to derivation of certain topological properties of SLFTs 
which are of independent interest as well as being preliminary to later results. In Section 3, we derive properties on pole-zero cancellation and McMillan degree via a state-space representation of an SLFT. Meanwhile, the problem of minimal stable linear fractional representations for a plant is considered and solved. In Section 4, we establish a one-to-one correspondence between the two sets of stabilizing controllers associated respectively with two plants related by an SLFT. An application of SLFTs in solving a standard $H^{\infty}$ control problem is also explored. Conclusions are drawn in Section 5.

\section{TOPOLOGICAL PROPERTIES}

In this section, we first give definitions and general properties of transformations of concern and then derive additional topological properties important for dealing with robust controller designs. The new properties are of independent interest, but also set the stage for later results.

A (right) stable linear fractional transformation (SLFT) $\mathscr{R}: R_{\mathrm{p}} \rightarrow R_{\mathrm{p}}$ is defined as

$$
G=\mathscr{R}(S) \triangleq(N+V S)(M+U S)^{-1}
$$

via

$$
Z=\left[\begin{array}{ll}
M & U \\
N & V
\end{array}\right] \in \mathbf{Z}
$$

where $R_{\mathrm{p}}$ denotes the set of proper rational matrices and $\mathbf{Z}$ is the set of all $2 \times 2$-block units $Z \in R H_{\infty}$ with the two diagonal block elements being an identity matrix at infinity and the $(1,2)$ block element being strictly proper. The restriction $Z \in \mathbf{Z}$ is imposed for simplicity of presentation and for making (1) well-defined for all $S \in R_{\mathrm{p}}$. If $Z^{-1}$ is compatibly partitioned as

$$
Z^{-1}=\left[\begin{array}{cc}
\tilde{V} & -\tilde{U} \\
-\tilde{N} & \tilde{M}
\end{array}\right]
$$

then the transformation $\mathscr{R}$ can be expressed in dual form

$$
\mathscr{R}(S)=(\tilde{M}+S \tilde{U})^{-1}(\tilde{N}+S \tilde{V})
$$

and the following Bezout identity holds

$$
\left[\begin{array}{cc}
\tilde{V} & -\tilde{U} \\
-\tilde{N} & \tilde{M}
\end{array}\right]\left[\begin{array}{ll}
M & U \\
N & V
\end{array}\right]=\left[\begin{array}{ll}
M & N \\
N & V
\end{array}\right]\left[\begin{array}{cc}
\tilde{V} & -\tilde{U} \\
-\tilde{N} & \tilde{M}
\end{array}\right]=I
$$

\section{Remark 2.1}

It can be readily established that the LFT $\mathscr{R}$ of the form (1) under (5) is a particular form of the following LFT

$$
\mathscr{F}(S)=J_{11}+J_{12} S\left(I-J_{22} S\right)^{-1} J_{21}
$$

with

$$
J \triangleq\left[\begin{array}{ll}
J_{11} & J_{12} \\
J_{21} & J_{22}
\end{array}\right]=\left[\begin{array}{cc}
N M^{-1} & \tilde{M}^{-1} \\
M^{-1} & -M^{-1} U
\end{array}\right]
$$


Conversely, any given LFT (6) with $J_{21}$ having a stable inverse can be rewritten in the form (1) with

$$
Z=\left[\begin{array}{ll}
M & U \\
N & V
\end{array}\right]\left[\begin{array}{cc}
J_{21}^{-1} & -J_{21}^{-1} J_{22} \\
J_{11} J_{21}^{-1} & J_{12}-J_{11} J_{21}^{-1} J_{22}
\end{array}\right]
$$

LFTs of the form (6) with $J_{i j}$ operators defined on a Hilbert space were dealt with by Redheffer in Reference 12. It is easy to see that under (7), $J$ is in $R H_{\infty}$ with $J_{21}$ a unit if $M$ is a unit in $R H_{\infty}$.

For our purposes, it is convenient to introduce a mapping $f$ from $\mathbf{Z}$ to the set of all SLFTs by defining

$$
f(Z)=\mathscr{R}
$$

A product $\mathscr{R}_{1} \mathscr{R}_{2}$ of two SLFTs $\mathscr{R}_{1}$ and $\mathscr{R}_{2}$ is defined by

$$
G=\mathscr{R}_{1} \mathscr{R}_{2}(S)=\mathscr{R}_{1}\left(\mathscr{R}_{2}(S)\right)
$$

Then it is straightforward to check that $f\left(Z_{1}\right) f\left(Z_{2}\right)=f\left(Z_{1} Z_{2}\right)$ for $Z_{1}, Z_{2} \in \mathbf{Z}$. By induction, one can prove

$$
f\left(Z_{1}\right) f\left(Z_{2}\right) \ldots f\left(Z_{n}\right)=f\left(Z_{1} Z_{2} \ldots Z_{n}\right)
$$

where $Z_{i} \in \mathbf{Z}, i=1, \ldots, n$. Consequently, it follows that

$$
\mathscr{R}^{-1}=f\left(Z^{-1}\right)
$$

These standard properties can be found in References 21,6 .

Before studying continuity of an SLFT, we first need to equip $R_{\mathrm{p}}$ with a topological structure. Given a $G_{0} \in R_{\mathrm{p}}$, let $\left(N_{0}, M_{0}\right)$ be any right coprime factorization (r.c.f.) of $G_{0}$ in $R H_{\infty}$, i.e. $G_{0}=N_{0} M_{0}^{-1}$. Then the set

$$
\mathbf{K}_{r}\left(N_{0}, M_{0} ; \varepsilon\right) \triangleq\left\{G=N M^{-1}: N, M \in R H_{\infty}, \quad \text { and }\left\|\left[\begin{array}{c}
N-N_{0} \\
M-M_{0}
\end{array}\right]\right\|<\varepsilon\right\}
$$

is called a basic neighbourhood of $G_{0}$ for any $\varepsilon>0$. It is known from Reference 17 that the collection of sets of the form (13), denoted by $\mathscr{K}_{\mathrm{r}}$, constitutes a base for a topology on $R_{\mathrm{p}}$, i.e., the so-called graph topology, which was first introduced and studied by Vidyasagar. In the same vein, it can be shown that the collection of sets $\mathbf{K}_{i}$ of the following form, denoted by $\mathscr{K}_{1}$, is also a base for a topology on $R_{\mathrm{p}}$ :

$$
\mathbf{K}_{i}\left(\tilde{N}_{0}, \tilde{M}_{0} ; \varepsilon\right) \triangleq\left\{G=\tilde{M}^{-1} \tilde{N}: \tilde{N}, \tilde{M} \in R H_{\infty}, \quad \text { and } \quad\left\|\left[\tilde{N}-\tilde{N}_{0} \quad \tilde{M}-\tilde{M}_{0}\right]\right\|<\varepsilon\right\}
$$

where $\left(\tilde{M}_{0}, \tilde{N}_{0}\right)$ is a left coprime factorization (l.c.f.) of $G_{0}$. Moreover, the two bases $\mathscr{K}_{\mathrm{r}}$ and $\mathscr{K}_{1}$ generate the same topology of $R_{\mathrm{p}}$. The main properties of SLFTs are collected in the following.

\section{Proposition 2.1}

Adopt the notation as above, there hold

(1) $\mathscr{R}=f(Z)$ with $Z \in \mathbf{Z}$ is a bijective and continuous map from $R_{\mathrm{p}}$ onto itself in the graph topology; so is its inverse.

(2) Given two units $Z_{1}, Z_{2} \in \mathbf{Z}, f\left(Z_{1}\right)=f\left(Z_{2}\right)$ if and only if there exists a scalar unit $\alpha \in R H_{\infty}$ such that $Z_{1}=\alpha Z_{2}$. 
(3) Given an SLFT $\mathscr{R}=f(Z)$ with

$$
Z=\left[\begin{array}{ll}
M & U \\
N & V
\end{array}\right]
$$

being a unit in $R H_{\infty}$, then for any $G \in \Omega \triangleq \mathbf{K}_{r}\left(N, M ; \varepsilon_{1}\right) \cup \mathbf{K}_{l}\left(\bar{N}, \tilde{M} ; \varepsilon_{2}\right)$ there exists a $S \in R H_{\infty}$ such that $G=\mathscr{R}(S)$, where

$$
\varepsilon_{1}=\frac{1}{\left\|\left[\begin{array}{ll}
\tilde{U} & \tilde{V}
\end{array}\right]\right\|} \quad \text { and } \quad \varepsilon_{2}=\frac{1}{\left\|\left[\begin{array}{l}
U \\
V
\end{array}\right]\right\|}
$$

Proof. See the Appendix.

As a direct consequence of (1) of Proposition 2.1, the following result states that an SLFT has the continuity-preserving property with respect to a parameter in a first-countable topological space. Recall that a first-countable topological space is one in which each point has a countable neighbourhood basis. ${ }^{17}$

\section{Corollary 2.1}

Suppose $\Lambda$ is a first-countable topological space. If $S_{\lambda}$, as a function of $\lambda$ mapping $\Lambda$ into $R_{\mathrm{p}}$, is continuous at $\lambda=\lambda_{0}$ in the graph topology, then so is $\mathscr{R}\left(S_{\lambda}\right)$. Likewise, if $G_{\lambda}$, as a function of $\lambda$ mapping $\Lambda$ into $R_{\mathrm{p}}$, is continuous at $\lambda=\lambda_{0}$ in the graph topology, so is $\mathscr{R}^{-1}\left(G_{\lambda}\right)$.

\section{Remark 2.2}

If one would like to use $\mathscr{R}(0)=N M^{-1}$ to represent an estimate of $G=\mathscr{R}(S)$, then (3) of Proposition 2.1 gives a quantitative description of how close $G$ is to $N M^{-1}$ so that $G$ can be expressed as an SLFT of $S \in R H_{\infty}$.

\section{POLE-ZERO CANCELLATION, McMILLAN DEGREE AND MINIMAL REPRESENTATION}

In this section, on the basis of astate-space representation of an SLFT we first reveal where pole-zero cancellations may occur for the state-space realization of an SLFT of a transfer matrix. Next, a generic McMillan degree relation between a transfer matrix and its SLFT is exhibited. Finally, we consider the problem of minimal representation of a plant as an SLFT of another plant such that the order of the original plant equals the sum of the orders of the SLFT and of the new plant. The necessary and sufficient conditions for solvability of such a problem are derived in terms of $(A, B)$-stable invariant subspaces.

Let the SLFT $\Re$ be given as in (1) with $Z$ a unit in $R H_{\infty}$ of the following state-space realization

$$
Z=\left[\begin{array}{ll}
M & U \\
N & V
\end{array}\right]=\left[\begin{array}{c|cc}
A & B_{1} & B_{2} \\
\hline C_{1} & D_{11} & D_{12} \\
C_{2} & D_{21} & D_{22}
\end{array}\right] \triangleq\left[\begin{array}{l}
C_{1} \\
C_{2}
\end{array}\right](s I-A)^{-1}\left[\begin{array}{ll}
B_{1} & B_{2}
\end{array}\right]+\left[\begin{array}{ll}
D_{11} & D_{12} \\
D_{21} & D_{22}
\end{array}\right]
$$


Assume

$$
S=\left[\begin{array}{c|c}
A_{s} & B_{S} \\
\hline C_{S} & D_{S}
\end{array}\right]
$$

satisfying that $\Delta \triangleq\left(D_{11}+D_{12} D_{S}\right)^{-1}$ exists. Since

$$
\left[\begin{array}{c}
M+U S \\
N+V S
\end{array}\right]=\left[\begin{array}{cc}
M & U \\
N & V
\end{array}\right]\left[\begin{array}{l}
I \\
S
\end{array}\right]=\left[\begin{array}{cc|c}
A & B_{2} C_{S} & B_{1}+B_{2} D_{S} \\
0 & A_{S} & B_{S} \\
\hline C_{1} & D_{12} C_{S} & D_{11}+D_{12} D_{S} \\
C_{2} & D_{22} C_{S} & D_{21}+D_{22} D_{S}
\end{array}\right]
$$

substituting into (1) yields

$$
G=\mathscr{R}(S)=\left[\begin{array}{cc|c}
A-\left(B_{1}+B_{2} D_{S}\right) \Delta C_{1} & B_{2} C_{S}-\left(B_{1}+B_{2} D_{S}\right) \Delta D_{12} C_{S} & \left(B_{1}+B_{2} D_{S}\right) \Delta \\
-B_{S} \Delta C_{1} & A_{S}-B_{S} \Delta D_{12} C_{S} & B_{S} \Delta \\
\hline-\left(D_{21}+D_{22} D_{S}\right) \Delta C_{1}+C_{2} & -\left(D_{21}+D_{22} D_{S}\right) \Delta D_{12} C_{S}+D_{22} C_{S} & \left(D_{21}+D_{22} D_{S}\right) \Delta
\end{array}\right]
$$

\section{Proposition 3.1}

Given the SLFT $\mathscr{R}(S)$. Let $\Gamma_{\mathrm{p}}$ and $\Gamma_{z}$ denote the sets of poles and of zeros of the state-space realization (16) of $Z$, respectively. Let $\Sigma_{\mathrm{c}}$ and $\Sigma_{\mathrm{o}}$ denote the sets of uncontrollable modes and of unobservable modes of the state-space realization (17), respectively. If the state-space realization $S$ is minimal and such that $\Delta\left(D_{11}+D_{12} D_{S}\right)^{-1}$ exists, then there holds

$$
\Sigma_{\mathrm{c}} \subset \Gamma_{\mathrm{p}} \text { and } \Sigma_{\mathrm{o}} \subset \Gamma_{\mathrm{z}}
$$

Proof.. See the Appendix.

In order to facilitate expositions in the sequel, we need the following definition.

\section{Definition 3.1}

Suppose the validity of a property depends on a vector $x$ in an $n$-dimensional Euclidean space. If there exists a finite system of polynomial equations in $n$-indeterminates such that at least a polynomial is nonzero and the property is true for all $x$ which are not a zero of the system of equations, then the property is said to hold generically with respect to $x$.

Quite obviously, if the property is generically true with respect to $x$, then the set of all $x$ for which the property holds is open and dense.

\section{Corollary 3.1}

With the same notation and hypotheses as in Proposition 3.1,

(i) The state-space realization (17) of $\mathscr{R}(S)$ is stabilizable and detectable with $\Sigma_{c} \cap \Sigma_{o}=0$ 
(ii) Let $\delta(\cdot)$ denote the McMillan degree and $m$ the number of columns of $B_{1}$. Assume that $\left(A, C_{1}\right)$ is observable. If

$$
\operatorname{rank}\left[\begin{array}{cccc}
\lambda I-A & B_{1} & B_{2} & 0 \\
C_{1} & D_{11} & 0 & D_{12}
\end{array}\right]=\delta(Z)+m, \quad \forall \lambda \in \mathbb{C}
$$

then there holds

$$
\delta(G)=\delta(Z)+\delta(S)
$$

generically with respect to minimal state-space realizations $\left(A_{S}, B_{S}, C_{S}, D_{S}\right)$ of $S$.

Proof. (i) is a direct consequence of Proposition 3.1 since both $Z$ and $Z^{-1}$ are stable. To prove (ii), set $\Psi \triangleq \Gamma_{\mathrm{c}} \cup \Gamma_{\mathrm{o}}$. It is apparent from Proposition 3.1 that (20) holds if the realization (17) does not contain any point in $\Psi$ as its mode. Write the state matrix of the realizations (17) in the form

$$
\begin{gathered}
{\left[\begin{array}{cc}
A-\left(B_{1}+B_{2} D_{S}\right) \Delta C_{1} & B_{2} C_{S}-\left(B_{1}+B_{2} D_{S}\right) \Delta D_{12} C_{S} \\
-B_{S} \Delta C_{1} & A_{S}-B_{S} \Delta D_{12} C_{S}
\end{array}\right]} \\
=\left[\begin{array}{cc}
A-B_{1} \Delta C_{1} & -B_{1} \Delta D_{12} C_{S} \\
0 & 0
\end{array}\right]+\left[\begin{array}{cc}
-B_{2} & 0 \\
0 & I
\end{array}\right]\left[\begin{array}{cc}
D_{S} & C_{S} \\
B_{S} & A_{S}
\end{array}\right]\left[\begin{array}{cc}
\Delta C_{1} & -\Delta D_{12} C_{S} \\
0 & I
\end{array}\right]
\end{gathered}
$$

It is easy to see that the observability of $\left(A, C_{1}\right)$ implies that of

$$
\left(\left[\begin{array}{cc}
A-B_{1} \Delta C_{1} & -B_{1} \Delta D_{12} C_{S} \\
0 & 0
\end{array}\right],\left[\begin{array}{cc}
\Delta C_{1} & -\Delta D_{12} C_{S} \\
0 & I
\end{array}\right]\right)
$$

It will be shown that the pair

$$
\left(\left[\begin{array}{cc}
A-B_{1} \Delta C_{1} & -B_{1} \Delta D_{12} C_{S} \\
0 & 0
\end{array}\right],\left[\begin{array}{cc}
-B_{2} & 0 \\
0 & I
\end{array}\right]\right)
$$

is controllable generically with respect to $\left(C_{S}, D_{S}\right)$. In fact, the pair (23) is controllable if and only if

$$
\operatorname{rank}\left[\lambda I-A+B_{1} \Delta C_{1} \quad B_{1} \Delta D_{12} C_{S} \quad B_{2}\right]=\delta(G), \quad \forall \lambda \in \mathbb{C}
$$

which is equivalent to

$$
\operatorname{rank}\left[\begin{array}{cccc}
\lambda I-A & -B_{2} & 0 & -B_{1} \\
C_{1} & 0 & D_{12} C_{S} & D_{11}+D_{12} D_{S}
\end{array}\right]=\delta(G)+m, \quad \forall \lambda \in \mathbb{C}
$$

under the constraint that $D_{11}+D_{12} D_{S}$ is invertible. Note that this constraint is satisfied generically for $D_{s}$. Making use of Lemma A.1 in the Appendix together with the assumption (19) yields that (25) holds generically with respect to $\left(C_{S}, D_{S}\right)$. Thus from Reference 4 , the lefthand side in (22), regarded as a closed-loop state matrix under a static output feedback, has no eigenvalues contained in $\Psi$ generically for $\left(A_{S}, B_{S}, C_{S}, D_{S}\right)$.

The following lemma is needed in the sequel. 


\section{Lemma 3.1}

Given a plant $G \in R_{p}$. A $2 \times 2$-block matrix

$$
Z=\left[\begin{array}{ll}
M & U \\
N & V
\end{array}\right]
$$

satisfies

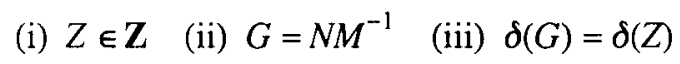

if and only if there exists a minimal realization

$$
\left[\begin{array}{l|l}
A & B \\
\hline C & D
\end{array}\right]
$$

of $G$ together with a stabilizing state feedback gain $F$ and a stabilizing output injection $H$ such that

$$
Z=\left[\begin{array}{c|cc}
A+B F & B & -H \\
\hline F & I & 0 \\
C+D F & D & I
\end{array}\right]
$$

Proof. The 'if' part is well known. Now it is assumed that the block matrix $Z$ satisfies (26) and has the following minimal realization

$$
Z=\left[\begin{array}{c|cc}
\bar{A} & \overline{B_{1}} & \overline{B_{2}} \\
\hline \bar{C}_{1} & I & 0 \\
C_{2} & D & I
\end{array}\right]
$$

Then, $A$ and $\bar{A}-B_{1} C_{1}-B_{2} C_{2}+B_{2} D C_{1}$ are stable. Setting

$$
A=\bar{A}-B_{1} C_{1}, \quad B=B_{1}, \quad C=C_{2}-D C_{1}, \quad F=C_{1}, \quad H=-B_{2}
$$

one can easily check that

$$
\left[\frac{A \mid B}{C} \mid D\right]
$$

is a minimal realization of $G$, that $F$ is a stabilizing state feedback gain and $H$ a stabilizing output injection, and that (27) holds.

\section{Remark 3.1}

For $Z$ given in (27), the conditions for (2) generically to hold amounts to the minimality of the system $(F, A, H)$.

(ii) of Corollary 3.1 tells us that given an SLFT $Z$ satisfying certain properties, the order of the image of a plant via this SLFT generically equals the sum of the orders of the plant and of $Z$. For a high-order plant $G$, it is often convenient to work first with its model $G_{0}$ and then with the frequency-shaped modelling error $S$. For example, as will be seen in the next section, the task of designing a stabilizing controller for $G$ can be accomplished by finding two respective stabilizing controllers for the model and the modelling error $S$. This method could divide a 
complex design problem into two or more simpler problems. Needless to say, the choice of model is important to success of the method in terms of efficiency and computational reduction. The acceptable choice should be one resulting in $S$ which has a lower order or can be approximated by a model of low order. Obviously, the really ideal case is where the complexity of $S$ is the plant's complexity minus the model's complexity. This prompts a question as to whether there exists a model for a given plant such that the order of the plant is the sum of the orders of the model and of the frequency-shaped modelling error, and how such a model can be constructed if it exists. To address this issue, we need to consider a minimal stable linear fractional representation, or MSLFR, for a plant, whose definition is given below.

\section{Definition 3.2}

A plant $G$ is said to have an MSLFR if there exists an SLFT $Z \in \mathbf{Z}$ and another plant $S \in R_{\mathrm{p}}$ such that $G=f(Z)(S)$ and

$$
\delta(Z), \delta(S)>0 \quad \text { and } \quad \delta(G)=\delta(Z)+\delta(S)
$$

$S$ will be called a pre-image of $G$.

It turns out that the problem of the existence of an MSLFR for a given plant is closely related to the problem of the existence of minimal factorization of a transfer function. The latter problem has been addressed and solved (see, for example, References 2 and 3 ). Recall that the factorization $R=R_{1} R_{2}$ is said to be minimal if $\delta(R)=\delta\left(R_{1}\right)+\delta\left(R_{2}\right)$ where $R, R_{1}, R_{2}$ are square rational matrices. Also, recall from Reference 18 that $\Phi$ is a stable invariant subspace of a pair $(A, B)$ if there exists $F$ such that $(A+B F) \Phi \subset \Phi$ and $A+B F$ is stable. The following result is used in the proof of the main result in this section.

\section{Lemma $3.2^{2,3}$}

Consider an $n \times n$ invertible transfer function matrix $G$ given by a minimal realization:

$$
G=\left[\begin{array}{l|l}
A & B \\
\hline C & D
\end{array}\right]
$$

Then there exists a minimal factorization for $G$ iff there exist independent subspaces $X_{1}$ and $X_{2}$ such that

$$
\text { (i) } A X_{1} \subset X_{1} \quad \text { (ii) }\left(A-B D^{-1} C\right) X_{2} \subset X_{2} \quad \text { (iii) } X_{1} \oplus X_{2}=X
$$

where $X$ denotes the state space.

\section{Theorem 3.1}

A given plant $G \in R_{\mathrm{p}}$ with a minimal realization (31) has an MSLFR if and only if there exist two stable invariant subspaces $X_{1}$ and $X_{2}$ associated with $(A, B)$ and $\left(A^{\tau}, C^{\tau}\right)$, respectively, such that

$$
X_{1} \oplus X_{2}^{\perp}=X
$$

where $X$ denotes the state space of the realization (31). 
Proof. Necessity: assume that there exists a nontrivial unit

$$
Z_{0}=\left[\begin{array}{ll}
M_{0} & U_{0} \\
N_{0} & V_{0}
\end{array}\right]
$$

and a plant $S \in R_{\mathrm{p}}$ such that

$$
G=\left(N_{0}+V_{0} S\right)\left(M_{0}+U_{0} S\right)^{-1} \quad \text { and } \quad \delta(G)=\delta\left(Z_{0}\right)+\delta(S)
$$

Since one can associate $S$ with a unit

$$
Z_{1}=\left[\begin{array}{ll}
M_{s} & U_{s} \\
N_{s} & V_{s}
\end{array}\right]
$$

where $N_{\mathrm{s}} M_{\mathrm{s}}^{-1}$ is an r.c.f. of $S$ and $\delta\left(Z_{1}\right)=\delta(S)$, it is seen that $G$ has the following r.c.f.

$$
G=\left(N_{0} M_{\mathrm{s}}+V_{0} N_{\mathrm{s}}\right)\left(M_{0} M_{\mathrm{s}}+U_{0} N_{\mathrm{s}}\right)^{-1}
$$

Define

$$
Z=\left[\begin{array}{ll}
M & U \\
N & V
\end{array}\right] \triangleq\left[\begin{array}{ll}
M_{0} & U_{0} \\
N_{0} & V_{0}
\end{array}\right]\left[\begin{array}{ll}
M_{s} & U_{s} \\
N_{s} & V_{s}
\end{array}\right]
$$

Since $\delta(Z)<\delta\left(Z_{0}\right)+\delta\left(Z_{1}\right)=\delta(G)$ and $\delta(Z)>\delta(G)$, it follows that $Z$ satisfies (26) and

$$
\delta(Z)=\delta\left(Z_{0}\right)+\delta\left(Z_{1}\right)
$$

Therefore, $Z=Z_{0} Z_{1}$ is a minimal factorization of $Z$. By Lemma 3.1, there exists a minimal realization

$$
\left[\begin{array}{l|l}
\mathscr{A} & \mathscr{B} \\
\hline \mathscr{C} & D
\end{array}\right]
$$

of $G$, a stabilizing state feedback gain $F$ and a stabilizing output injection $H$ such that

$$
\left[\begin{array}{c|cc}
\mathscr{A}+\mathscr{B} F & \mathscr{B} & -H \\
\hline F & I & 0 \\
\mathscr{C}+D F & D & I
\end{array}\right]
$$

is a minimal realization of $Z$. By Lemma 3.2, there exist subspaces $X_{1}$ and $X_{2}$ such that

$$
\begin{array}{lll}
\text { (i) }(\mathscr{A}+\mathscr{B} F) Y_{1} \subset Y_{1} & \text { (ii) }(\mathscr{A}+H \mathscr{C}) Y_{2} \subset Y_{2} & \text { (iii) } Y_{1} \oplus Y_{2}=X
\end{array}
$$

Evidently, (i) in the above implies that $Y_{1}$ is a stable invariant subspace of $(\mathscr{A}, \mathscr{B})$ while (ii) implies that $Y_{2}^{\perp}$ is that of $\left(\mathscr{A}^{\tau}, \mathscr{C}^{\tau}\right)$. Since $(\mathscr{A}, \mathscr{B}, \mathscr{C}, D)$ and $(A, B, C, D)$ are minimal realizations of $G$, there exists a similarity transformation $T$ such that

$$
\mathscr{A}=T^{-1} A T, \quad \mathscr{B}=T^{-1} B, \quad \mathscr{C}=C T
$$

Let $X_{1}=T Y_{1}$ and $X_{2}=\left(T^{-1}\right)^{\mathrm{r}} Y_{2}^{\perp}$. Then it is not hard to see that $X_{1}$ and $X_{2}$ are two stable invariant subspaces $X_{1}$ and $X_{2}$ associated with $(A, B)$ and $\left(A^{\tau}, C^{\tau}\right)$, respectively, and satisfies (33).

Sufficiency: choose $F$ and $H$ such that $A+B F$ and $A+H C$ are stable, and that
(i) $(A+B F) X_{1} \subset X_{1}$
(ii) $\left(A^{\tau}+C^{\tau} H^{\tau}\right) X_{2} \subset X_{2}$
(iii) $X_{1} \oplus X_{2}^{1}=X$ 
This implies by Lemma 3.2 that the $Z$ with the minimal realization (27) has a minimal factorization, say $Z=Z_{1} Z_{2}$ where $Z_{i} \in R_{\mathrm{p}}$. Since $Z$ is a unit and there is no pole-zero cancellation between its two factors $Z_{1}$ and $Z_{2}, Z_{1}$ and $Z_{2}$ are units in $R H_{\infty}$. Furthermore, there is no loss of generality in assuming that $Z_{i} \in \mathbf{Z}, i=1,2$. Letting $S \cong N_{2} M_{2}^{-1}$ leads to $G=f\left(Z_{1}\right)(S)$, which implies

$$
\delta(G) \leqslant \delta\left(Z_{1}\right)+\delta(S) \leqslant \delta\left(Z_{1}\right)+\delta\left(Z_{2}\right)
$$

From the minimality of the factorization $Z=Z_{1} Z_{2}$ and the above inequalities, it follows that $\delta(G)=\delta\left(Z_{1}\right)+\delta(S)$. In this way, the proof of the theorem is completed.

In fact, the above result can be generalized as follows.

Theorem 3.2

Given a plant $G \in R_{\mathrm{p}}$ with a minimal realization (31). There exist a series of SLFTs $Z_{1}, Z_{2}, \ldots, Z_{k}$ and $S$ such that

$$
G=f\left(Z_{1}\right) f\left(Z_{2}\right) \ldots f\left(Z_{k}\right)(S) \quad \text { and } \quad \delta(G)=\sum_{i=1}^{k} \delta\left(Z_{i}\right)+\delta(S)
$$

if and only if there exist $(k-1)$ pairs of stable invariant subspaces $X_{i}$ and $Y_{i}$ associated with $(A, B)$ and $\left(A^{\tau}, C^{\tau}\right)$, respectively, nested as follows:

$$
\{0\} \subset X_{1} \subset \ldots \subset X_{k-1} \subset X \text { and }\{0\} \subset Y_{1} \subset \ldots \subset Y_{k-1} \subset X
$$

and such that

$$
X_{i} \oplus Y_{i}^{\perp}=X, \quad i=1, \ldots, k
$$

where $X$ denotes the state space.

Proof.. The proof is omitted.

Corollary 3.2

With the same assumption and notation as in Theorem 3.1, if $(A, B)$ and $\left(A^{\tau}, C^{r}\right)$ have a common stable invariant subspace, then $G$ has an MSLFR.

\section{Remark 3.2}

The proof of Theorem 3.1 apparently provides a two-step procedure to construct an MSLFR. The first step is to find a pair of stable invariant subspaces $X_{1}$ and $X_{2}$ of $(A, B)$ and $\left(A^{\tau}, C^{r}\right)$, respectively, which satisfy (33). In the SISO case, this reduces to finding a nontrivial stable invariant subspace of $(A, B)$ since $(A, B)$ and $\left(A^{\tau}, C^{\tau}\right)$ have the same set of stable invariant subspaces. An iterative algorithm to compute the supremal $(A, B)$-stable invariant subspace contained in a subspace is given in Reference 18. The second step involves constructing a minimal factorization of a unit in $\mathbf{Z}$. For algorithms to perform a minimal factorization of a rational matrix, see, for example, Reference 3. 


\section{STABILIZATION AND $H^{\infty}$-OPTIMIZATION}

In this section we first describe how the set of all stabilizing controllers for a plant will change when the plant is transformed by an SLFT. It turns out that another SLFT can be induced from the original SLFT to establish a one-to-one correspondence between two respective sets of all stabilizing controllers associated with the plant and its image. Next, we show how a standard $H^{\infty}$ cminimization problem for a plant can be approximately broken into two individual subproblems associated respectively with two models of lower order.

As usual, a plant transfer matrix $G \in R_{\mathrm{p}}$ is said to be stabilized by a controller $C \in R_{\mathrm{p}}$ iff

$$
H(G, C) \triangleq\left[\begin{array}{cc}
I & -G \\
-C & I
\end{array}\right]^{-1}
$$

exists and belongs to $R H_{\infty}$. The set of all the stabilizing controllers for $G$ is denoted by $\mathbf{S}(G)$. Now assume that $G_{\lambda}$ is in $R_{\mathrm{p}}$ for all $\lambda$ in a first-countable topological space $\Lambda$. Then according to Vidyasagar, ${ }^{17} G_{\lambda}$ is defined to be robustly stabilized by a controller $C \in R_{\mathrm{p}}$ at $\lambda=\lambda_{0}$ if there exists a neighbourhood $\mathbf{N}$ of $\lambda_{0}$ such that $C \in \mathbf{S}\left(G_{\lambda}\right), \forall \lambda \in \mathbf{N}$, and moreover, $H\left(G_{\lambda}, C\right)$ is continuous as a map from $\Lambda$ to $R_{\mathrm{p}}$ at $\lambda_{0}$ in the graph topology. It is known again from Reference 17 that $C \in R_{\mathrm{p}}$ robustly stabilizes $G_{\lambda}$ at $\lambda=\lambda_{0}$ iff $C \in \mathbf{S}\left(G_{\lambda_{0}}\right)$ and $G_{\lambda}$ is continuous at $\lambda=\lambda_{0}$ in the graph topology.

Given a transfer matrix $G \in R_{\mathrm{p}}$ of the form

$$
G=f(Z)(S)
$$

where $S$ is a transfer matrix in $R_{\mathrm{p}}, Z \in \mathbf{Z}$ is of the form (2), and $f$ is the mapping from $\mathbf{Z}$ to the set of SLFTs, as defined in Section 2. For the given $Z$, we introduce three associated matrices $\Gamma_{i}(Z), i=1,2,3$, defined by

$$
\begin{gathered}
\Gamma_{1}(Z) \triangleq\left[\begin{array}{ll}
0 & I \\
I & 0
\end{array}\right]\left[\begin{array}{ll}
M & U \\
N & V
\end{array}\right]\left[\begin{array}{ll}
0 & I \\
I & 0
\end{array}\right] \\
\Gamma_{2}(Z) \triangleq\left[\begin{array}{cc}
0 & I \\
-I & 0
\end{array}\right] Z^{-1}\left[\begin{array}{cc}
0 & -I \\
I & 0
\end{array}\right] \\
\Gamma_{3}(Z) \triangleq\left[\begin{array}{cc}
0 & -N \\
-U & 0
\end{array}\right] \Gamma_{2}(Z)
\end{gathered}
$$

It will be seen in the proof of the next result that given a closed-loop system, the transfer function of the new closed-loop system resulting from a suitable transformation on the plant and the controller can be related to the original transfer function in terms of the above-defined matrices. Quite obviously, $\Gamma_{1}(Z)$ and $\Gamma_{2}(Z)$ are two units in $R H_{\infty}$ while $\Gamma_{3}(Z)$ belongs to $R H_{\infty}$. In addition, $\Gamma_{1}(Z)$ is in $Z$ iff the $(2,1)$ block element of $Z$ vanishes at infinity. It is also useful to introduce an induced SLFT $\mathscr{R}^{*}$ of $\mathscr{R}$ by defining

$$
\mathscr{R}^{*}=f\left(\Gamma_{1}(Z)\right)
$$

Then $\mathscr{R}^{*}$ is well-defined whenever $\Gamma_{1}(Z)$ is in $\mathbf{Z}$. One can easily check that $\left(\mathscr{R}^{-1}\right)^{*}=\left(\mathscr{R}^{*}\right)^{-1}$.

Before stating a simple one-to-one correspondence between $\mathbf{S}(G)$ and $\mathbf{S}(S)$ via the induced SLFT $\mathscr{R}^{*}$, we indicate that $\mathbf{S}(G)$ is an open subset of $R_{\mathrm{p}}$ in the graph topology for any given $G \in R_{\mathrm{p}}$. By duality, the set of plants which are stabilizable by a given controller is also open in $R_{\mathrm{p}}$. 


\section{Proposition 4.1}

Consider two transfer matrices $G$ and $S$ related by $G=\mathscr{R}(S)=f(Z)(S)$. Assume $Z$, $\Gamma_{1}(Z) \in \mathbf{Z}$. Then $C=\mathscr{R}^{*}(Q)$ is a continuous bijective mapping from $\mathbf{S}(S)$ onto $\mathbf{S}(G)$ in the graph topology.

Proof. Since by (1) of Proposition 2.1, $f(Z)$ is continuous and bijective from $R_{\mathrm{p}}$ onto itself in the graph topology for any $Z \in \mathbf{Z}$, it suffices to show that $C=\mathscr{R}^{*}(Q)$ is in $\mathbf{S}(G)$ iff $Q$ is in $\mathbf{S}(S)$. To do this, first note that

$$
C=\mathscr{R}^{*}(Q)=(U+M Q)(V+N Q)^{-1}
$$

If $H(S, Q)$ exists, a simple calculation leads to

$$
H(G, C)=\Gamma_{1}(Z) H(S, Q) \Gamma_{2}(Z)+\Gamma_{3}(Z)
$$

By considering $\mathscr{R}^{-1}=f\left(Z^{-1}\right)$, one similarly has that if $H(G, C)$ exists, then

$$
H(S, Q)=\Gamma_{1}\left(Z^{-1}\right) H(G, C) \Gamma_{2}\left(Z^{-1}\right)+\Gamma_{3}\left(Z^{-1}\right)
$$

In view of the fact that both $\Gamma_{1}(Z)$ and $\Gamma_{2}(Z)$ are unimodular in $R H_{\infty}$, it follows from $(51)$ and (52) that $H(G, C)$ exists and belongs to $R H_{\infty}$ iff so does $H(S, Q)$.

\section{Corollary 4.1}

With the same assumptions and notation as in Proposition 4.1, further, assume that $G_{\lambda}=\mathscr{R}\left(S_{\lambda}\right)$ where $G_{\lambda}$ and $S_{\lambda}$ depend on $\lambda$ in a first-countable topological space. Then, $C$ robustly stabilizes $G_{\lambda}$ at $\lambda=\lambda_{0}$ iff $Q=\left(\mathscr{R}^{*}\right)^{-1}(C)$ robustly stabilizes $S_{\lambda}$ at $\lambda=\lambda_{0}$.

In view of the property (11), the above two results can be easily generalized as follows.

\section{Corollary 4.2}

Let a plant transfer matrix $G_{0}$ be represented in the following recursive form

$$
G_{i}=\left(N_{i}+V_{i} G_{i+1}\right)\left(M_{i}+U_{i} G_{i+1}\right)^{-1}, \quad i=0,1, \ldots, n-1
$$

where

$$
Z_{i}=\left[\begin{array}{cc}
M_{i} & U_{i} \\
N_{i} & V_{i}
\end{array}\right] \in \mathbf{Z} \quad \text { and } \quad \Gamma_{1}\left(Z_{i}\right) \in \mathbf{Z}, \quad i=0,1, \ldots, n-1
$$

They any controller $C_{0} \in \mathbf{S}\left(G_{0}\right)$ can be recursively parameterized by

$$
C_{i}=\left(U_{i}+M_{i} C_{i+1}\right)\left(V_{i}+N_{i} C_{i+1}\right)^{-1}, \quad i=0,1, \ldots, n-1
$$

with $C_{n} \in \mathbf{S}\left(G_{n}\right)$. Moreover, if it is further assumed that $G_{i}, i=0,1, \ldots, n-1$, depend on $\lambda$ in a first-countable topological space, then $C_{0}$ robustly stabilizes $G_{0}$ at $\lambda=\lambda_{0}$ iff $C_{n}$ robustly stabilizes $G_{n}$ at $\lambda=\lambda_{0}$.

\section{Remark 4.1}

The recursive fractional representation (53) of the plant $G_{0}$ of ten arises owing to successive identification for $G_{i}, i=0, \ldots, n-1$. Corollary 4.2 suggests that a multi-controller strategy 
could be proposed for a plant with a recursive fractional representation. This together with internal stability properties is investigated in Reference 20.

We now turn to consider the basic block diagram depicted in Figure 1, where $G$ is the generalized plant of the form

$$
G=\left[\begin{array}{ll}
G_{11} & G_{12} \\
G_{21} & G_{22}
\end{array}\right]
$$

and $C$ is the controller. The standard $H^{\infty}$ control problem for Figure 1 is to find a controller $C$ to minimize the $H^{\infty}$-norm of the transfer function from $w$ to $z$

$$
\mathscr{F}(G, C)=G_{11}+G_{12} C\left(I-G_{22} C\right)^{-1} G_{21}
$$

under the constraint that $C$ stabilizes $G$. In practice, if a low-order model $G_{22}^{0}$ of $G_{22}$ is already available, then one may first try to solve the $H^{\infty}$ optimal control problem for the nominal generalized plant

$$
G_{0} \triangleq\left[\begin{array}{ll}
G_{11} & G_{12} \\
G_{21} & G_{22}^{0}
\end{array}\right]
$$

As will be noted, in certain situations $\left\|\mathscr{F}\left(G_{0}, C_{0}\right)\right\|_{\infty}$ is actually the minimal achievable $H^{\infty}$ norm of the closed-loop transfer function associated with the plant. However, when the obtained 'optimal' controller $C_{0}$ is applied to the true generalized plant $G$, a totally unsatisfactory closedloop $H^{\circ}$ performance may result if $G_{22}^{0}$ is not a good enough approximate to $G_{22}$. The question we are interested in is how to further design an additional controller solely based on some modelling error between $G_{22}$ and $G_{22}^{0}$ so that the $H^{\infty}$-norm of the transfer function from $w$ to $z$ can be brought as close to that of $\mathscr{F}^{\prime}\left(G_{0}, C_{0}\right)$ as possible.

To this end, let $N M^{-1}$ and $U V^{-1}$ be stable coprime factorizations of $G_{22}^{0}$ and $C_{0}$, respectively, with the Bezout identity (5) being satisfied. Then, $G_{22}$ can be represented as a stable linear fractional transformation of some transfer function $S$, i.e.,

$$
G_{22}=(N+V S)(M+U S)^{-1}
$$

Here, $S$ is actually a frequency-shaped mismatch between $G_{22}$ and $G_{22}^{0}$. Now consider an augmented controller arrangement shown in Figure 2, where $J_{0}$ is the augmented controller of $C_{0}$ in the form

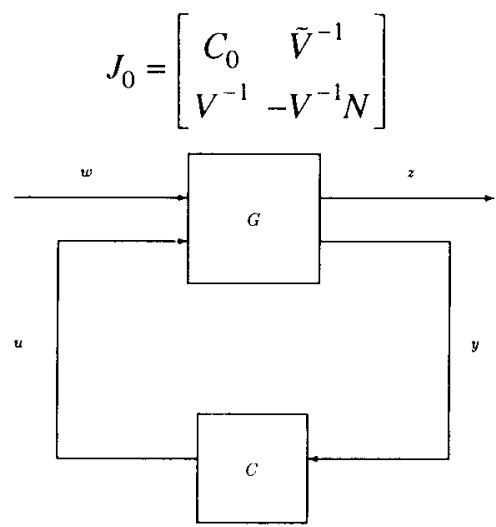

Figure 1. Basic block diagram. 


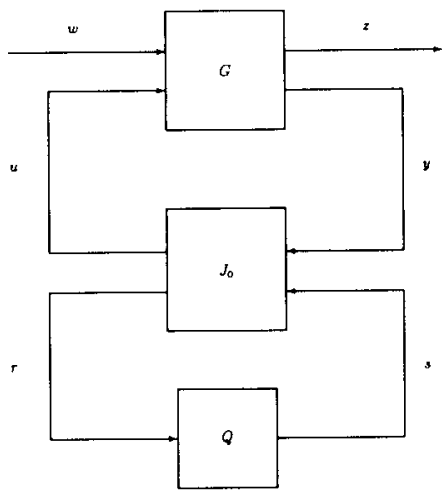

Figure 2. Augmented block diagram.

and $Q$ is the additional controller. Obviously, such an arrangement is reduced to the standard one in Figure 1 if $Q$ is set to zero. Also note that $S$ is the transfer function from $s$ to $r$ and thus should be identifiable under reasonable conditions. In view of this, we will assume that $S$ is known.

The following main result of this section shows that minimizing the difference between the transfer function from $w$ to $z$ and the 'ideal' transfer function $\mathscr{F}\left(G_{0}, C_{0}\right)$ by means of the additional controller $Q$ selection approximates to a standard $H^{\infty}$ controller design task. Moreover, this design is purely based on the frequency-shape modelling error $S$.

\section{Theorem 4.1}

Consider the augmented block diagram in Figure 2 together with (56) and (58)-(60). Assume that $G_{12}$ and $G_{21}$ are stable. Let $T_{w z}$ denote the transfer function from $w$ to $z$. Define $\alpha$ independent of $S$ as

$$
\alpha=\left\|G_{12}\left[\begin{array}{ll}
U & M
\end{array}\right]\right\|_{\infty}\left\|\left[\begin{array}{c}
\tilde{M} \\
U
\end{array}\right] G_{21}\right\|_{\infty}
$$

Then there holds

$$
\left\|T_{w z}-\mathscr{F}\left(G_{0} C_{0}\right)\right\|_{\infty} \leqslant \alpha\|\mathscr{F}(P, Q)\|_{\infty}
$$

where $P$ is a generalized error model defined by

$$
P=\left[\begin{array}{ll}
P_{11} & P_{12} \\
P_{21} & P_{22}
\end{array}\right]=\left[\begin{array}{ll}
{\left[\begin{array}{ll}
0 & S \\
0 & 0
\end{array}\right]\left[\begin{array}{l}
S \\
I
\end{array}\right]} \\
{\left[\begin{array}{ll}
I & S
\end{array}\right]} & S
\end{array}\right]
$$

Proof. First note that the transfer function from $y$ to $u$ is simply the image of $Q$ by the SLFT corresponding to $\Gamma_{1}(Z)$, namely,

$$
C(Q)=(U+M Q)(V+N Q)^{-1}
$$

Making use of the relation (51) yields

$$
C(Q)\left[I-G_{22} C(Q)\right]^{-1}=\left[\begin{array}{ll}
0 & I
\end{array}\left[\Gamma_{1}(Z) H(S, Q) \Gamma_{2}(Z)+\Gamma_{3}(Z)\right]\left[\begin{array}{l}
I \\
0
\end{array}\right]=\left[\begin{array}{ll}
U & M
\end{array}\right] H(S, Q)\left[\begin{array}{l}
\tilde{M} \\
\tilde{U}
\end{array}\right]-U \tilde{M}\right.
$$


from which it follows that

$$
\begin{aligned}
& T_{w z}=\mathscr{F}(G, C(Q))=G_{11}+G_{12} C(Q)\left[I-G_{22} C(Q)\right]^{-1} G_{21}
\end{aligned}
$$

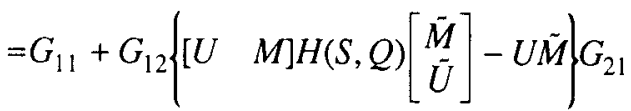

Setting $S$ and $Q$ to zero in the above immediately gives

$$
\mathscr{F}\left(G_{0}, C_{0}\right)=G_{11}+G_{12} M \tilde{U} G_{21}
$$

Hence, one has

$$
T_{w z}-\mathscr{F}\left(G_{0}, C_{0}\right)=G_{12}[U \quad M][H(S, Q)-I]\left[\begin{array}{c}
\tilde{M} \\
\tilde{U}
\end{array}\right] G_{21}
$$

This apparently implies (62) upon noticing that $\mathscr{F}(P, Q)=H(S, Q)-I$.

\section{Remark 4.2}

Although the generalized plant and its model may have the almost same optimal achievable $H^{\infty}$ performance in some cases, the fact that a controller $C_{0}$ minimizes $\left\|F_{F}\left(G_{0}, C\right)\right\|_{\infty}$ does not necessarily imply that it also minimizes $\|\mathscr{F}(G, C)\|_{\infty}$. Rather, if the $C_{0}$ is applied to the true plant $G$, the resulting closed-loop $H^{\infty}$ performance could be very poor, as will be demonstrated in the coming example.

In the light of Theorem 4.1, a multistage procedure for designing a suboptimal $H^{\infty}$ controller could be suggested as follows.

Step 1 Get a low-order nominal model $G_{22}^{0}$ for $G_{22}$.

Step 2 Design an optimal $H^{\infty}$ controller $C_{0}$ for the nominal generalized plant $G_{0}$.

Step 3 Get an estimate $S_{0}$ of the stable linear fractional transformation $S=\left(-\tilde{N}+\bar{M} G_{22}\right)$ $\times\left(\tilde{V}-\tilde{U} G_{22}\right)^{-1}$ where $\tilde{M}^{-1} \tilde{N}$ and $\tilde{V}^{-1} \tilde{U}$ are l.c.f.s of $G_{22}^{0}$ and $C_{0}$, respectively.

Step 4 Design an optimal $H^{\infty}$ controller $Q$ for the generalized plant $P_{0}$ associated with $S_{0}$ via (63).

Step 5 If the single-loop diagram in Figure 1 is adopted, generate the SLFT $C=(U+M Q)$ $\times(V+N Q)^{-1}$ which is the desired suboptimal $H^{\infty}$ controller; if the two-loop diagram in Figure 2 is adopted, augment the generalized controller $J_{0}$ resulting from $C_{0}$ by the additional controller $Q$.

\section{Remark 4.3}

The above design method seems particularly useful and effective in the case where $S$ is unstable and/or large in $H^{\infty}$-norm. Although the order of the SLFT $S$ may be high, using its estimate of fairly low order can often result in a satisfactory performance, as shown in the following example. The order of the overall suboptimal controller using the procedure is the sum of the orders of $G_{0}$ and $S_{0}$, which tends to be less than the order of the true plant $P$.

\section{Remark 4.4}

By Proposition 4.1, the designed controller stabilizes the original system if and only if $S$ is 
stabilized by $Q$ which is stabilizing $S_{0}$. Therefore, if there is no modelling error between $S$ and $S_{0}$, then the closed-loop stability is guaranteed. Using (3) of Proposition 2.1, one can easily derive a quantitative measure of how small the modelling error is between $S$ and $S_{0}$ so that $Q$ can stabilize not only $S_{0}$ but also $S$. Details about stabilization of the multiple controller structure can be found in Reference 20.

\section{Remark 4.5}

It is worth pointing out some differences between the multistage approach proposed here and the separation approach in Reference 5 to the $H^{\infty}$ control problem. First, the separation approach is used to reduce a general output feedback problem to an equivalent output estimation problem with a plant of the same order, while the multistage approach is aimed at decomposing an $H^{\infty}$ problem into two subproblems, each of which is associated with a lower-order plant and thus can be more easily solved using the separation principle. In passing, we would like to mention that there is a great incentive to break a complex problem into smaller pieces from a software engineering viewpoint. Second, the separation approach can lead to an optimal $H^{\infty}$ controller whereas the other usually yields a suboptimal controller. Therefore, if the order of the original plant is low enough, there is no advantage in using the multistage approach. Last, owing to its nature, the multistage approach could have potential applications in the context of successive identification and control in the presence of uncertain dynamics in the plant.

Finally, we present an example to illustrate the proposed design method for the standard $H^{\infty}$ control problem.

Consider the true generalized plant

$$
G=\left[\begin{array}{ll}
G_{11} & G_{12} \\
G_{21} & G_{22}
\end{array}\right]
$$

where

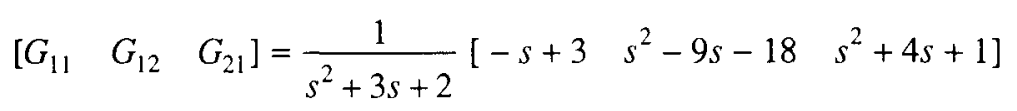

and

$$
\left.G_{22}=\left[\begin{array}{rrrrrr}
0.0 & 1.0 & 0.0 & 0.0 & 0.0 & 0.0 \\
-2.0 & -3.0 & 0.4 & 7.2 & 0.0 & 0.0 \\
0.0 & 0.0 & -5.3 & -9.7 & 2.2 & -1.0 \\
0.0 & 0.0 & -5.3 & -14.0 & 4.8 & -2.4 \\
-2.7 & -2.7 & -2.3 & -43.0 & 0.0 & 1.0 \\
-3.6 & -3.6 & -3.0 & -10.0 & -30.0 & -20.0
\end{array}\right]\left[\begin{array}{r}
0.0 \\
1.0 \\
0.0 \\
0.0 \\
6.0 \\
8.0
\end{array}\right]\right]
$$

We choose the model $G_{22}^{0}$ of $G_{22}$ as follows

$$
G_{22}^{0}=\left[\begin{array}{rr|r}
{\left[\begin{array}{rr}
0.0 & 1.0 \\
-2 \cdot 0 & -3 \cdot 0
\end{array}\right]} & {\left[\begin{array}{l}
0 \cdot 0 \\
1.0
\end{array}\right]} \\
\hline\left[\begin{array}{ll}
-2 & -5
\end{array}\right] & 0
\end{array}\right]
$$


The resulting modelling error $G_{22}-G_{22}^{0}$ is depicted in Figure 3. Then for the nominal plant

$$
G_{0}=\left[\begin{array}{ll}
G_{11} & G_{12} \\
G_{21} & G_{22}^{0}
\end{array}\right]
$$

an optimal $H^{\infty}$ controller is found to be

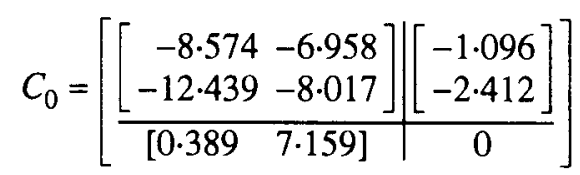

The magnitude plot of $\mathscr{F}\left(G_{0}, C_{0}\right)$ is depicted in Figure 4 where $\left\|\mathscr{F}\left(G_{0}, C_{0}\right)\right\|$ appears to be less than $2 \cdot 7$. (In fact, $\left\|\mathscr{F}\left(G_{0}, C\right)\right\|$ is always greater than $2 \cdot 6$ for any stabilizing $C$.) When this $C_{0}$, which turns out to stabilize $G$, is applied to $G$, the $H^{\infty}$-norm of the closed-loop transfer function $T_{w z}$ reaches above 30 (see Figure 5) although its achievable minimum is the same as $\left\|\mathscr{F}\left(G_{0}, C_{0}\right)\right\|$, i.e., $2 \cdot 7$ or so. This is because of unmodelled dynamics.

To design the additional controller $Q$, we choose an SLFT

$$
\left[\begin{array}{cc}
\tilde{V} & -\tilde{U} \\
-\tilde{N} & \tilde{M}
\end{array}\right]
$$

based on $G_{0}$ and $C_{0}$. Thereby the frequency-shaped modelling error $S$ of order 10 can be generated. But, we only use its second-order balanced-truncation model to form the generalized

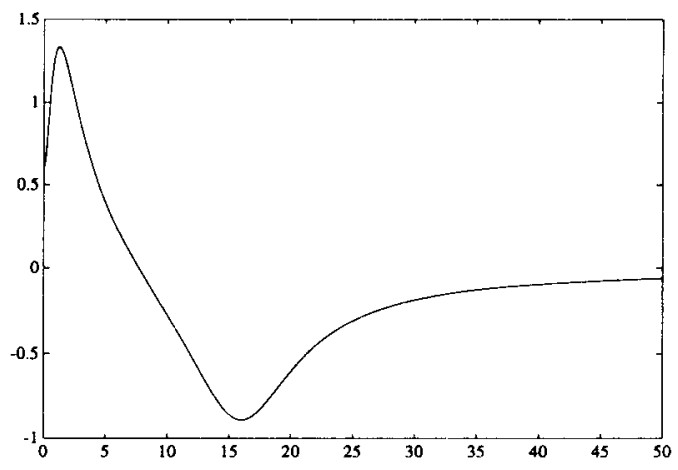

Figure 3. Magnitude plot of $G_{22}-G_{22}^{0}$

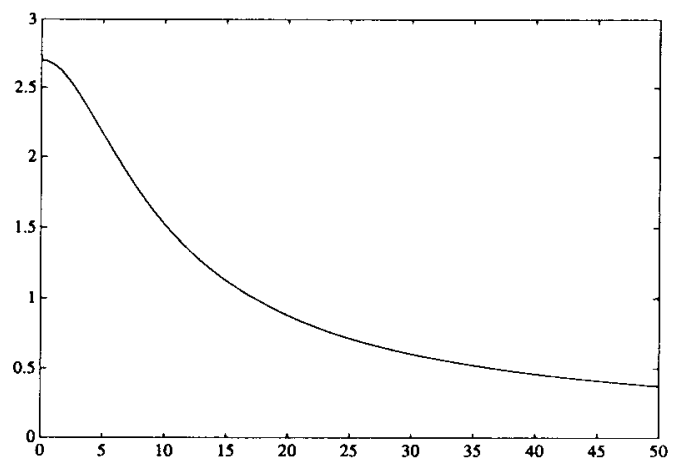

Figure 4. Magnitude plot of $\mathscr{F}\left(G_{0}, C_{0}\right)$ 


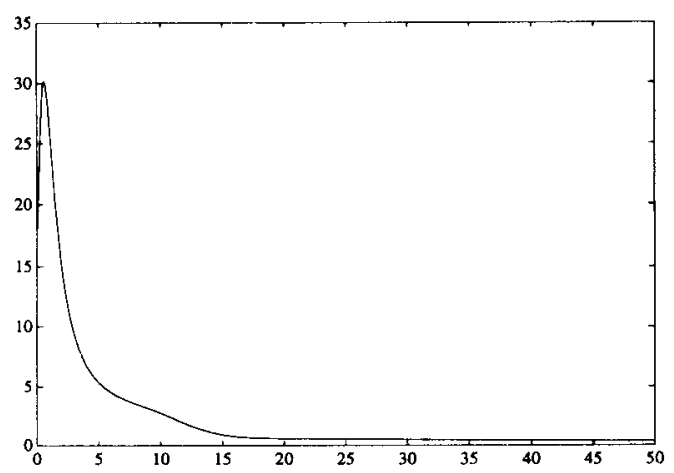

Figure 5. Magnitude plot of $T_{w:}$ associated with $C_{0}$

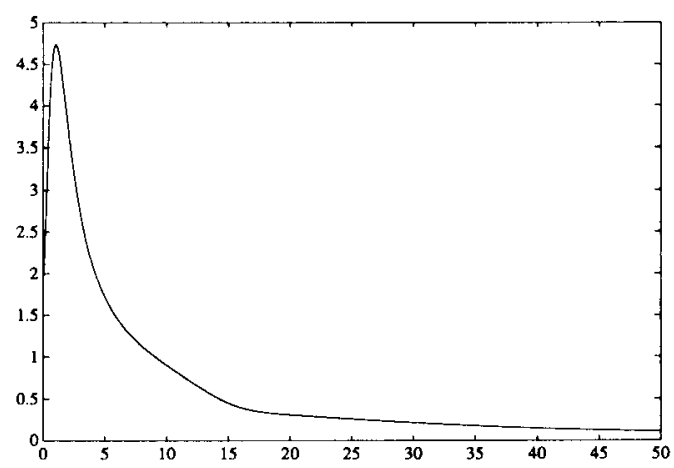

Figure 6. Magnitude plot of $T_{w: z}$ associated with $C(Q)$

error model $P$ via (63). Then an optimal $H^{\infty}$ controller $Q$ for $P$ is given by

$$
Q=\left[\begin{array}{ll}
{\left[\begin{array}{ll}
-3.1067 & -7.0481 \\
-7.0481 & -30.7053
\end{array}\right]} & {\left[\begin{array}{l}
-0.4283 \\
-4.2655
\end{array}\right]} \\
\hline\left[\begin{array}{ll}
-0.4283 & -4.3655]
\end{array}\right] & 0
\end{array}\right]
$$

Construct the controller $C(Q)=(U+M Q)(V+N Q)^{-1}$ which is of order 4 and evidently stabilizes $G$, and apply it to $G$. Not unexpectedly, the $H^{\infty}$-norm of $T_{w z}$ is dramatically reduced to 4.7 or so; see Figure 6.

\section{CONCLUSIONS}

We have considered a stable linear fractional transformation mapping $R_{\mathrm{p}}$ to itself. Some properties of the transformation on pole-zero cancellation and McMillan degree have been obtained by virtue of its state-space representation. A one-to-one correspondence between the two sets of stabilizing controllers respectively for two plants related by an SLFT has been established. However, the main contributions of this paper are the following. First, we have derived the necessary and sufficient conditions for the existence of a minimal stable linear fractional representation for a given plant in terms of stable invariant subspaces. This result answers the question as to when there exists a model for a given plant such that the complexity of the plant may be distributed precisely on the model and the resulting frequency-shaped modelling error. If such a model exists, a stabilization problem for the plant can be broken into the problem of stabilizing successively two models whose orders add up to the order of the 
original plant. Second, it has been shown that a standard $H^{\infty}$ control problem can be sectioned into two individual subproblems via an SLFT. On the basis of this, a new multistage method of designing a suboptimal $H^{\infty}$ controller is proposed and its effectiveness has been demonstrated by an example.

\section{ACKNOWLEDGEMENTS}

This work was in part supported by Boeing (BCAC) and DSTO Australia.

\section{APPENDIX}

\section{Proof of Proposition 2.1}

(1) The proof is quite standard and omitted.

(2) Clearly, it suffices to show that given a $Z \in \mathbf{Z}, \mathscr{R}=f(Z)$ is an identity mapping, i.e., $\mathscr{R}(S)=S$, $\forall S \in R_{\mathrm{p}}$ iff $Z_{0}$ is of the form $Z=z I$, where $z$ is a scalar unit in $R H_{\infty}$. Sufficiency is obvious on noting that under $Z=z I$ then $N=U=0$ and $M=V=z I$. As for necessity, since $\mathscr{R}(S)=S$ for all $S \in R_{\mathrm{p}}$, taking $S=0$ yields $N=0$. Consequently, $R(S)=S$ is equivalent to

$$
V S-S M=S U S
$$

We now establish that (73) holds for all $S \in R_{\mathrm{p}}$ only if $U=0$ and $V=M=z I$ for some scalar unit $z \in R H_{\infty}$. First of all, $U$ must be equal to zero. Otherwise, there is no loss of generality in assuming

$$
U=\left[\begin{array}{cc}
\operatorname{diag}\left\{u_{1}, \ldots, u_{r}\right\} & 0 \\
0 & 0
\end{array}\right]
$$

where $u_{1}, \ldots, u_{r}$ are the invariant factors of $U$. Compatibly, partition $M, V$ into

$$
M=\left[\begin{array}{ll}
M_{11} & M_{12} \\
M_{21} & M_{22}
\end{array}\right] \text { and } V=\left[\begin{array}{ll}
V_{11} & V_{12} \\
V_{21} & V_{22}
\end{array}\right]
$$

and in particular, choose

$$
S=\left[\begin{array}{cc}
\alpha I & 0 \\
0 & 0
\end{array}\right]
$$

with $\alpha \in R_{\mathrm{p}}$. Then (73) implies

$$
\alpha\left(V_{11}-M_{11}\right)=\alpha^{2} \operatorname{diag}\left\{u_{1}, \ldots, u_{r}\right\}
$$

Quite evidently, the above relation cannot hold for all scalar $\alpha \in R_{\mathrm{f}}$. This contradiction shows $U=0$. In this way, (73) becomes $V S-S M=0$, or equivalently, $\left(I \otimes V-M^{\prime} \otimes I\right) S=0$. Since this holds for all $S \in R_{\mathrm{p}}$, one can see that $I \otimes V-M^{\prime} \otimes I=0$, from which it follows that $V=M=z I$ for some scalar $z \in R H_{\infty}$. Further, $z$ must be a unit since $Z$ is a unit.

(3) Let $G$ be in $\mathbf{K}_{r}\left(N, M ; \varepsilon_{1}\right)$. Then there exist r.c.f. $\left(N_{1}, M_{1}\right)$ and l.c.f. $\left(\tilde{M}_{1}, \bar{N}_{1}\right)$ of $G$ in $R H_{\infty}$ such that

$$
\left\|\left[\begin{array}{c}
N_{1}-N \\
M_{1}-M
\end{array}\right]\right\|<\varepsilon_{1}
$$

Since

$$
\bar{V} M_{1}-\tilde{U} N_{1}=I+\tilde{V}\left(M_{1}-M\right)-\tilde{U}\left(N_{1}-N\right)
$$

(7.4) yields that $\tilde{V} M_{1}-\tilde{U} N_{1}$ is a unit in $R H_{\infty}$, which in turn implies that

$$
S \triangleq\left(\tilde{M} N_{1}-\tilde{N} M_{1}\right)\left(\tilde{V} M_{1}-\tilde{U} N_{1}\right)^{-1} \in R H_{\infty}
$$


It can be further verified that $(M+U S)^{-1}$ exists and $S$ satisfies $\mathscr{R}(S)=G$. Using a similar argument, one can reach the same conclusion for $G \in \mathbf{K}_{i}\left(\tilde{N}, \tilde{M} ; \varepsilon_{2}\right)$ as for $G \in \mathbf{K}_{\mathrm{r}}\left(N, M ; \varepsilon_{1}\right)$.

\section{Proof of Proposition 3.1}

Let $\lambda_{0} \in \Sigma_{\mathrm{c}}$. Then there exists a nonzero vector $x^{\mathrm{\tau}}=\left[\begin{array}{ll}x_{1}^{\tau} & x_{2}^{\tau}\end{array}\right]$ such that

$$
\left[\begin{array}{ll}
x_{1}^{\tau} & x_{2}^{\tau}
\end{array}\right]\left[\begin{array}{ccc}
\lambda_{0} I-A+\left(B_{1}+B_{2} D_{S}\right) \Delta C_{1} & -B_{2} C_{S}+\left(B_{1}+B_{2} D_{S}\right) \Delta D_{12} C_{S} & \left(B_{1}+B_{2} D_{S}\right) \Delta \\
B_{S} \Delta C_{1} & \lambda_{0} I-A_{S}+B_{S} \Delta D_{12} C_{S} & B_{S} \Delta
\end{array}\right]=0
$$

which is evidently equivalent to

$$
\left[\begin{array}{ll}
x_{1}^{\tau} & x_{2}^{\tau}
\end{array}\right]\left[\begin{array}{ccc}
\lambda_{0} I-A & -B_{2} C_{S} & B_{1}+B_{2} D_{S} \\
0 & \lambda_{0} I-A_{S} & B_{S}
\end{array}\right]=0
$$

This implies that $x^{\tau}{ }_{1}\left(\lambda_{0} I-A\right)=0$. Noting $x \neq 0$, one can claim that $x_{1} \neq 0$ because, otherwise, (75) would result in $x^{\mathrm{r}}{ }_{2}\left[\lambda_{0} I-A_{S} \quad B_{S}\right]=0$, which is contradictory to the assumption that $\left(A_{S}, B_{S}\right)$ is controllable. In this way, it follows that $\lambda_{0}$ is an eigenvalue of $A$. On the other hand, if $\lambda_{0}$ is an uncontrollable mode of multiplicity $k$, i.e., there exist $k$ linearly independent vectors $x_{i}^{r}=\left[\begin{array}{ll}x^{r}{ }_{i 1} & x^{r}{ }_{i 2}\end{array}\right], i=1, \ldots, k$, the above reasoning tells us that $x_{i 1}, \ldots, x_{k 1}$ are linearly independent eigenvectors of $A$ associated with $\lambda_{0}$. Hence, it is concluded that $\Sigma_{\mathrm{c}} \subset \Gamma_{\mathrm{p}}$. To prove that $\Sigma_{0} \subset \Gamma_{2}$, we need to note that the inverse of $T \triangleq D_{22}-\left(D_{21}+D_{22} D_{S}\right) \Delta D_{12}$ exists as the $(2,2)$-block element of the block matrix

$$
\left[\begin{array}{ll}
D_{11}+D_{12} D_{S} & D_{12} \\
D_{21}+D_{22} D_{S} & D_{22}
\end{array}\right]^{-1}
$$

Thus, the polynomial matrix

$$
\left[\begin{array}{cc}
\lambda I-A+\left(B_{1}+B_{2} D_{S}\right) \Delta C_{1} & -B_{2} C_{S}+\left(B_{1}+B_{2} D_{S}\right) \Delta D_{12} C_{S} \\
B_{S} \Delta C_{1} & \lambda I-A_{S}+B_{S} \Delta D_{12} C_{S} \\
-\left(D_{21}+D_{22} D_{S}\right) \Delta C_{1}+C_{2} & -\left(D_{21}+D_{22} D_{S}\right) \Delta D_{12} C_{S}+D_{22} C_{S}
\end{array}\right]
$$

is equivalent to

$$
\left[\begin{array}{cc}
\lambda I-A+\left(B_{1}+B_{2} D_{S}\right) \Delta C_{1}+\left[B_{2}-\left(B_{1}+B_{2} D_{S}\right) \Delta D_{12}\right] T^{-1} & 0 \\
B_{S} \Delta C_{1} & \lambda I-A_{S}+B_{S} \Delta D_{12} C_{S} \\
T^{-1}\left[C_{2}-\left(D_{21}+D_{22} D_{S}\right) \Delta C_{1}\right] & C_{S}
\end{array}\right]
$$

which turns out to equal

$$
\left[\begin{array}{cc}
\lambda I-A+\left[\begin{array}{ll}
B_{1} & B_{2}
\end{array}\right]\left[\begin{array}{ll}
D_{11} & D_{12} \\
D_{21} & D_{22}
\end{array}\right]^{-1}\left[\begin{array}{l}
C_{1} \\
C_{2}
\end{array}\right] & 0 \\
B_{S} \Delta C_{1} & \lambda I-A_{S}+B_{S} \Delta D_{12} C_{S} \\
T^{-1}\left[C_{2}-\left(D_{21}+D_{22} D_{S}\right) \Delta C_{1}\right] & C_{S}
\end{array}\right]
$$

In view of the equivalence between (76) and (77), and the fact that $\Gamma_{2}$ consists of eigenvalues of the matrix

$$
A-\left[\begin{array}{ll}
B_{1} & B_{2}
\end{array}\right]\left[\begin{array}{ll}
D_{11} & D_{12} \\
D_{21} & D_{22}
\end{array}\right]^{-1}\left[\begin{array}{l}
C_{1} \\
C_{2}
\end{array}\right]
$$

one can prove $\Sigma_{0} \subset \Gamma_{2}$ using the same argument as for $\Sigma_{\mathrm{c}} \subset \Gamma_{\mathrm{p}}$. 
Lemma $A .1^{19}$

Suppose $\{D(s), M(s), N(s)\}$ are $m \times n, m \times p$ and $m \times q$ polynomial matrices, with rank $D(s)>m-p-\varepsilon$ where $\varepsilon$ is some nonnegative integer. Then for almost all $q \times p$ constant matrices $K$,

$$
\operatorname{rank}[D(s) M(s)+N(s) K] \geqslant m-\varepsilon, \quad \forall s \in \mathbb{C}
$$

if and only if both of the following conditions hold for all $s \in \mathbb{C}$ :

(a) $\operatorname{rank}[D(s) M(s) N(s)] \geqslant m-\varepsilon$

(b) $\operatorname{rank} D(s) \geqslant m-p-\varepsilon$

\section{REFERENCES}

1. Antoulas, A. C., 'On recursiveness and related topics in linear systems', IEEE Trans. Automatic Control, AC-31, $1121-1135$ (1986).

2. Bart, H., I. Gohberg, M. A. Kaashoek and P. Van Dooren, 'Factorizations of transfer functions', SIAM J. Control Opt., 18, 675-696 (1980).

3. Van Dooren, P. and P. Dewilde, 'Minimal cascade factorization of real and complex rational transfer matrices', IEEE Trans. Circuits and Systems, CAS-28, 390-400 (1981).

4. Davison, E. J. and S. H. Wang, 'Properties of linear time-invariant multivariable systems subject to arbitrary output and state feedback', IEEE Trans. Automatic Control, AC-18, 24-32 (1973).

5. Doyle, J. C., K. Glover, P. P. Khargonekar and B. A. Francis, 'State-space solutions to standard $H_{2}$ and $H_{\infty}$ control problems', IEEE Trans. Automatic Control, AC-34, 831-847 (1989).

6. Dym, H., J-Contractive Matrix Functions, Reproducing Kernel Hilbert Spaces and Interpolation, Vol.71 of the Regional Conference Series in Mathematics, American Mathematical Society, Providence, RI, 1989.

7. Francis, B. A., A Course in $H_{\infty}$ Control Theory, Vol. 88 of Lecture Notes in Control and Information Systems, Springer Verlag, New York, 1987.

8. Glover, K. and D. McFarlane, 'Robust stabilization of normalized coprime factor plant description with $H_{\infty}$ bounded uncertainty', IEEE Trans. Automatic Control, AC-34, 821-830 (1989).

9. Glover, K. and D. McFarlane, 'Robust stabilization of normalized coprime factor plant description with $H_{\infty}$ ' bounded uncertainty', IEEE Trans. Automatic Control, AC-34, 821-830 (1989).

10. Kimura, H., 'Conjugation, interpolation and model-matching in $H_{\infty}$ ', Int. J. Control, 49, 269-307 (1989).

11. Limebeer, D. J. N. and Y. S. Hung, 'An analysis of the pole-zero cancellations in $H^{\infty}$-optimal control problems of the first kind', SIAM J. Control Opt., 25, 1457-1493 (1987).

12. Redheffer, R. M., 'On a certain linear fractional transformation', J. Math \& Phys., 39, 269-286 (1960).

13. Saeks, R. and J. Murray, 'Fractional representation, algebraic geometry, and the simultaneous stabilization problem', IEEE Trans. Automatic Control, AC-27, 895-903 (1982).

14. Tay, T. T. and J. B. Moore, 'Enhancement of fixed controllers via adaptive disturbance estimate feedback', Automatica, 27, 39-53 (1991).

15. Tay, T. T., J. B. Moore and R. Horowitz, 'Indirect adaptive techniques for fixed controller performance enhancement', Int. J. Contr., 50, 1941-1960 (1990).

16. Tsai, M. C, and I. Postlethwaite, 'On J-lossless coprime factorizations and $H_{\infty}$ control', Int. J. Robust and Nonlinear Control, 1, 47-68 (1991).

17. Vidyasagar, M., Control System Synthesis: a Factorization Approach, Cambridge, MA, MIT Press, 1985.

18. Wonham, W. M., Linear Multivariable Control: a Geometric Approach, Springer Verlag, New York, 1979.

19. Yan, W. Y. and R. R. Bitmead, 'Decentralized control of multi-channel systems with direct control feedthrough', Int. J. Control, 49, 2057-2075 (1989).

20. Yan, W. Y. and J. B. Moore, 'A multiple controller structure and design strategy with stability analysis', Automatica, 28, 1239-1244 (1992).

21. Yong, N. J., 'Linear fractional transformations in rings and modules', Linear Algebra and its Applications, 56, $251-290$ (1984). 